\title{
Study on Opportunities, Challenges, and Improvement of Moral Education in Colleges and Universities Under Information Age
}

\author{
Qi ZHOU* \\ Shanghai Jian Qiao University, Shanghai, China \\ Email: shzq18@126.com \\ ${ }^{*}$ Corresponding author
}

\begin{abstract}
Keywords: informatization, college and university students, moral education
Abstract. With the advent of the informatization era, people's work, study and life have been closely connected with electronic devices, and data is everywhere today. Under the development of information technology, the thoughts, behavior, morality and belief of college and university students are undergoing tremendous changes. This paper focuses on analyzing moral education in colleges and universities under information age, exploring opportunities and challenges faced by moral education and providing research thought for improvement.
\end{abstract}

\section{Introduction}

The 21st century is an era of informatization. Research on exploring and developing the moral education under the environment of informatization is an important subject for all colleges and universities. The advancement of information technology has freed college students from their ivory towers. They can understand and explore the society and acquire various information resources about learning and living with a positive, open and free mindset through the Internet. This kind of unprecedented convenience has provided conditions for ideological emancipation and innovation of students in colleges and universities. However, information outside the ivory tower is various and complex, which also brings many problems to those college and university students whose minds and thoughts are not mature enough. The moral education in colleges and universities is confronted with challenges, but also opportunities and improvement there.

\section{Influence on Moral Education under Informatization Age}

\subsection{Reconstruction of Moral Cognitive Structure of College University Students}

The most important influence of information age on moral education in university is to reconstruct the moral cognitive structure of students. Before being admitted to colleges and university, students mostly receive traditional moral education with a single cognitive structure. However, under the background of information age, the free transfer of information has allowed a single cognitive structure to spread, then pluralistic social values begin to form. Based on information transmission through Internet, students are more willing to believe in the information that they can see. They avoid habitually thinking about all received information through traditional moral cognition, so they are easily affected by various information with inducibility. The incomplete information brought by massive fragmented data has interfered with the moral cognition of college and university students. At the same time, it has impacted on the traditional cognitive structure that has been formed but not yet solidified. It is an invasion and occupation of the original moral knowledge and moral experience, which brings a reconstructing of the moral cognitive structure of college and university students.

\subsection{Moral Emotion Transformation of College and University Students}

Under the background of the information age, college and university students are allowed to quickly receive data and information due to abundant external information, convenient channels for 
information acquisition, multiple ways of information transmission and precise information transmission spread channels. As a result, the moral emotion of college students is transformed in a short period of time, and teachers are not given sufficient time to provide guidance for those students, which is not conducive to the development of moral education in colleges and universities. At present, information has evolved from a single text form to multiple forms such as audio, video and virtual reality experiences. Students can instantly gain a deep moral experience; thereby it will deepen the basis for emotional transformation. In addition, the emotional transformation results of college and university students are becoming increasingly diverse. In the information age, a large number of internet cultures have been spawned. The era is coupled with various channels of mobile information channels, so the results of emotional transformation of college and university students can be quickly spread out, which fully superimposes the rate of information transmission. This results in rapid ferment of students' emotions which become more difficult to be controlled and guided. Consequently, the social influence continues to deepen.

\subsection{Abnormal Variations in Moral Behavior of College and University Students}

In general, behavioral variation is almost manifested as a large subjective pursuit in the subconscious. Most of the college students have not undergone enough social trainings, and their psychology and physiology are relatively immature. Meanwhile, their emotions are coupled with the depression caused by various reasons. Once there are lack of scientific guidance and management, their emotions and behaviors will easily burst out in a short time, which breaks the original moral cognition structure. This brings fluctuations in their psychology and physiology, which affects the way of thinking and causes moral behavior variations. College and university students have been in an information explosion environment for a long time, and they are easily affected by various kinds of information. With the lapse of time, a great moral pressure if formed in their hearts. If this pressure can't be properly channeled and released, the moral behavior of college students will change abnormally. Moreover, the richer the information environment, the greater the chance of abnormal variation of moral behavior.

\section{Opportunities of Moral Education Under Informatization Age}

\subsection{Statistics and Analysis of Moral Behavior of College and University Students}

After entering the information age, the behaviors of college and university students are often collected and sorted as data. With the help of professional big data processing software, all behaviors can be classified and stored by accurate analysis. All moral factors contained in various behaviors of students will be performed with breaking analysis. This convenience maximizes the use of data to analyze the moral status of students. By using information technology, it is simulated to process of campus moral education can be simulated, the results data of moral education can be calculated, and correction suggestions for moral education can be provided in time, which is helpful for statistics, monitoring and analysis of college students' moral behavior.

\subsection{Meeting the Individualized Needs of Moral Learning}

Under the background of the information age, there are individualized design for moral education courses in colleges and universities. Based on data technology, some well-directed teaching plans and assessment plans can be formulated for different levels and needs of college and university students. Through data technology, the current behavior and moral emotional status of college students can be comprehended, which is helpful to differentiate the moral status and moral behavior enthusiasm of college students, so as to promote supplementary information that is helpful to moral education. It combines reality to provide participation channels of social activities about moral education, so as to improve the proactivity of learning of college students. 


\subsection{A Carrier Provided for Reform of Moral Education}

Traditional moral education mainly relies on text and pictures. Books, videos and television works are as the main carriers. But whichever channel is to be chosen for spread for those carries, there must be certain hysteresis. This hysteresis is not in accordance with the current fast-paced learning and living status. In the information age, the channels to receive information are expanding. While the timeliness of information is gradually increasing, the data attribute of behavior is also increasing. All actions of college and university students can be counted and divided into different types of data which is stored in the big data center. This storage will help for statistical analysis of a large number of common moral behaviors of students. These data computed by data model can be used as an important basis for content reform, method innovation and mechanism improvement in moral education. It can also be used as channels and terminal carriers of moral education, allowing college and university students to receive information anytime and anywhere and allowing students to accept various moral education, which makes up for the weaknesses of traditional moral education with single form its poor expandability and pertinence.

\section{Challenges of Moral Education Under Informatization Age}

\subsection{Challenges from Increased Difficulties in Moral Education}

Compared with the traditional moral education, the biggest challenge for college and university moral education in the information age is to the moral education becomes harder. In recent years, the students' beliefs, moral consciousness, moral identity and behavior have undergone tremendous changes with the development of information technology. In the information age, network data has brought a huge amount of information, which has made students' consciousness occupied by various values. Meanwhile, because of rebellious psychology caused by age, experience and other reasons, the moral consciousness and moral belief of college and university students are easily invaded by radical negative values, later occurrence of anti-conventional behavior.

\subsection{Challenges from Data Collection and Use in Moral Education}

In the background of information age, there is a mutual function of data. College and university students can obtain a large amount of information through information technology. Colleges and universities can also collect behavioral data of students through information technology. However, colleges and universities also face challenges in data collection, analysis and use. First, they need to establish a campus information network that can collect data anytime and anywhere. Second, they need to set up a suitable cloud computing center that can handle data tasks such as about life, learning, moral emotions and behavior patterns. Finally, cooperation among universities is needed to form a large sample database of students' behaviors. Only in this way can the practical effects of data in the information age on university moral education be truly exerted.

\subsection{Higher Requirements for Teachers' Professionality in Moral Education}

In the background of information age, there are higher requirements for teacher's abilities. Except traditional abilities as university teachers, they also need to advance with the times in accordance with the steps of information age. First, teachers need to master certain capabilities of data mining and they are able to process data through cloud computing thus to grasp moral behaviors of college students. Second, teachers need to have abilities of education innovation, and they can make timely adjustments to teaching content, and they can make reasonable attempts on new teaching models, and they can improve the teaching evaluation mechanism, in order to truly improve teaching efficiency and teaching quality. Third, teachers need to be equipped with profound theoretical knowledge. Only by combining the emotional needs of with traditional culture, can the course become a rich mine for students to draw better moral values. 


\section{Improvement of Moral Education Under Informatization Age}

\subsection{Grasping Characteristics of Moral Education under Informatization Age}

In the education of moral level of college and university students, the students should change the traditional educational concepts and educational cognition. Based on the current situation of moral education in the information age, they need to grasp the following characteristics of moral education.

First, the development characteristics of moral cognition of students in the information age must be grasped. In the information age, students' moral cognition has gradually changed from the past form of experience accumulation to logical reasoning. Students can process and analyze various received information and a critical and independent thinking mode has been formed. Therefore, we must analyze the moral behavior according to its moral cognition characteristics of college and university students.

Second, the times characteristics of informatization. Under the information environment, the information explosion has become normal in society. Teachers need to understand the current development status of information technology. Through information technology, they should analyze and sort out the moral behavior of college and university students, and they should use the data network to teach the Chinese excellent traditional moral culture for their students. The purpose is to make students shape their own behavior principles and moral standards.

Third, it is needed to grasp the growth characteristics of college and university students. In the information age, the students are generally in a state that their thinking consciousness is inconsistent with their psychological consciousness. They have ability of storing a considerable amount of information, but they are lack of the corresponding ability to handle and process information. Therefore, on the one hand, colleges and university should get with the thinking consciousness of students and they should use a relatively equal way of thinking when communicating with students. On the other hand, they should also have certain auxiliary measures in moral education to help students to solve their psychological pressure and psychological problems.

\subsection{Establishment of Multidimensional Education Mode Based on Informatization}

Most of moral behaviors and moral tendencies of college and university students are hidden in their daily behaviors. During management, colleges and universities can collect a large amount of behavior data of students, but these data have obvious characteristics of fragmentation. This requires colleges and universities to establish multidimensional education modes based on informatization. Colleges and universities need to establish data collection networks that rely on the internet and life data collection centers. Then, the data is processed through cloud computing or third-party platforms. Meanwhile, mathematical models are used to analyze the moral behaviors hidden behind the data. The most important thing is to adopt highly targeted and personalized education modes for college and university students of different levels and states. Moreover, the data analysis results are used to establish a warning mechanism for college and university students 'moral behavior. It is helpful to actively guide students' moral behaviors and moral thoughts, and timely eliminate negative thoughts through moral education.

\subsection{Improvement of Multiple Evaluation Mechanisms for Moral Education}

In the information age, moral education in colleges and universities needs to constantly improve its evaluation mechanism. Traditional moral education mostly adopts the duality evaluation mode including daily moral behavior and examination. However, this evaluation mode has features of certain contingency and speculation, which cannot objectively and truly reflect the moral level of college and university students. Accordingly, teachers need to innovate the evaluation mode and supplement the evaluation content. For example, the data collected through the campus data networks can be processed and analyzed; the moral behaviors of college and university students can 
be scored; and the daily behaviors collected can be scientifically and quantitatively evaluated; and the evaluation results can be regularly fed back to college students in the form of information. The purpose is to push forward college and university students to learn and provide corresponding behavioral guidance and interpretation of moral evaluation. It is needed to regularly analyze and sort out the feedback information about the content and form of moral education from students. Colleges and universities should continuously adjust and improve the teaching process, and enhance the quality of moral education.

\section{Conclusion}

In the background of information age, the moral education of college and university students is no longer confined to classrooms with limited space, but it has expanded into college and university life with illimitable space. Information technology provides an innovative driving force for moral education. It is an important guarantee to make the moral education of great scientification, standardization, individuation and precision. However, we should also pay close attention to various potential problems brought by information technology, such as internet security, information protection and accountability for moral issues. Therefore, on the one hand, the capabilities of teachers in colleges and universities should be extended, the awareness of big data should be strengthened, the innovative educational modes should be encouraged, and the evaluation mechanism should be improved. On the other hand, it is necessary to speed up the construction of hardware and security, which is significant to cultivate more talents in the new era.

\section{References}

[1] Zhaoliang Gu. Research on Ideological and Political Education Innovation of College and University Students from the Perspective of Individualized Learning [D]. Thesis of Southwest Jiaotong University, 2017

[2] Jing Wang. Research on moral education of college and university students in the era of big data [D]. Thesis of Southwest University, 2015

[3] Min Zhao. Research on Moral Education Innovation of College and University Students in the Perspective of New Media [D]. Thesis of Shandong University, 2012

[4] Yanya Luo. Research on Moral Education for College and University Students from the Perspective of Network Communication—A Review of Research on Network Communication and Moral Cultivation of College and University Students[J]. Research in Educational Development, 2019 (10): 2

[5] Jie Deng. Necessity and Strategy for Strengthening Moral Education in Network Communication of College and University Students [J]. Modern Communication, 2019 (8): 134-135

[6] Yanyan Yao, Yuxuan Lin, Jingsong Niu. A Study on the Innovation of Moral Education of College and University Students in the New Media Era_A Review of New Exploration of Moral Education of Contemporary College and University Students[J]. Journalism Lover, 2019 (2): 107

[7] Guanjun Jiang, Jianguo Zhang. Research on Moral Education of College and University Students in the "Best Beauty" Perspective_—A Case Study of Campus Cultural Activities Based on "Finding Touching around us " [J]. New Exploration of Ideological and Political Education, 2016: 257-267

[8] Lijie Jiang, Jia Li. Exploration of Moral Education of College and University students from the Perspective of Big Data [J]. China's Rural Education, 2018 (24): 10, 38 